\title{
Gobernabilidad y presupuestos sensibles al género: estudios y experiencias en África ${ }^{1}$
}

\author{
Governance and Gender \\ Responsive Budgeting: \\ Studies and Experiences in Africa
}

\section{CARMEN ASCANIO SÁNCHEZ*}

Resumen: En los últimos decenios se han venido desarrollando numerosas investigaciones sobre presupuestos con enfoque de género, por lo que en la actualidad se cuenta con una importante producción, tanto teórica como empírica. Este artículo analiza algunas experiencias puestas en marcha en seis países africanos, de especial interés por estar directamente relacionadas con áreas prioritarias y planes de desarrollo nacional, y lideradas desde organismos y agencias de desarrollo internacional, con el apoyo de diversos agentes sociales. Se plantea aquí la relevancia de esta perspectiva de género, del enfoque comprensivo y comparativo que conlleva, así como los resultados de dicha comparación en el contexto africano y las recomendaciones más notables.

Palabras clave: gobernabilidad; presupuestos de género; indicadores de género; África; políticas de igualdad de género.

Recepción: 19 de diciembre de 2016. / Aceptación: 16 de marzo de 2017.

* Universidad de La Laguna, cascanio@ull.edu.es

${ }^{1}$ Este trabajo se enmarca en el proyecto "Gobernabilidad, género y desarrollo. Estrategias de fortalecimiento, cooperación y difusión en África del Oeste”, Agencia Española de Cooperación Internacional al Desarrollo, Ministerio de Asuntos Exteriores y de Cooperación de España, dirigido por la autora (2011-2013). Una reflexión inicial se presentó en el $8^{\circ}$ Congreso Ibérico de Estudios Africanos en 2012. Véase un resumen en Carmen Ascanio Sánchez, "Gobernabilidad y presupuestos sensibles al género. Estado de la cuestión en África”, Pueblos. Revista de información y debate, 3 de agosto de 2012, pp. 1-2. 
Abstract: In recent decades, much research has been conducted on the topic of gender-sensitive budgeting, giving rise to a substantial body of both theoretical and empirical work. This paper analyzes a range of such projects carried out in six different African countries, which are of particular interest given their direct link to priority areas and plans for national development, and the fact that they have been spearheaded by international development agencies and bodies and conducted with the assistance of a range of social partners. We examine the relevance of a gender-based perspective and of the comprehensive and comparative approach it embodies, as well as presenting the results of comparative work in the African context and the main recommendations which come out of it.

Key words: governance; gender budgeting; gender indicators; Africa; gender equality policies.

La asignación de recursos presupuestarios para los distintos grupos sociales es, sin duda alguna, un indicador del interés de los gobiernos en materia de equidad. ${ }^{2}$ Los denominados presupuestos sensibles al género (PSG) han centrado parte de los debates relacionados con gobernabilidad y buenas prácticas y, por tanto, con la promoción de la equidad, la participación, el pluralismo y la transparencia. En el mundo se cuenta con numerosos estudios que aportan experiencias, enfoques, herramientas y evaluaciones, ${ }^{3}$ y que también han explicitado las amenazas del binomio equidad/desarrollo, entre ellas la corrupción, la violencia y la pobreza.

Este artículo plantea la trascendencia de la perspectiva de género ${ }^{4}$ y de los PSG en el contexto mundial. Expone, asimismo,

${ }^{2}$ Recientes estudios desde la disciplina económica lideran este enfoque; véase Tindara Addabbo, "Gender Budgeting in the Capability Approach. From Theory to Evidence", presentación invitada en la WiSE: Women in Scotland's Economy Series Conference 'Counting on Women-Gender, Care and Economics', Glasgow Caledonian University, 24-26 de mayo de 2011.

${ }^{3}$ Debbie Budlender y Rhonda Sharp, con Kerri Allen, How to do a Gendersensitive Budget Analysis: Contemporary Research and Practice, Canberra-Londres, Commonwealth Secretariat-Australian Agency for International Development, 1998.

${ }^{4}$ Entendemos por género un concepto multifacético basado en la percepción sociocultural de las diferencias entre los sexos y que, entre muchos otros elementos, 
las trayectorias de este enfoque presupuestario en seis países africanos. Su elección se basa en dos hechos: primero, que han sido los pioneros en desarrollar este tipo de experiencias, iniciadas -aproximadamente- a mediados y finales de la década de 1990; segundo, que cuentan con publicaciones internacionales que informan de dichos procesos. Esto último es determinante para realizar un estado de la cuestión o, al menos, avanzar en él. En el caso africano, las publicaciones científicas son escasas y gran parte de lo que se conoce son informes de carácter general, evaluativo y, en ocasiones, meramente informativo. En este sentido, se consideraron las dificultades de un análisis sistematizado o comparativo de los PSG en África, sea por áreas, liderazgos, agentes implicados o resultados.

Por lo anterior, el objetivo de este artículo es contribuir a un inicial estado de la cuestión sobre la implementación de estas políticas de igualdad de género en las agendas de los gobiernos de estos países pioneros, ofreciendo un marco global del proceso, señalando similitudes y diferencias en su desarrollo y, por último, sistematizando avances, retrocesos y recomendaciones. La metodología se basó en un análisis de los principales enfoques e intervenciones en PSG en el mundo, en la experiencia propia en varios proyectos realizados y, principalmente, en una revisión y análisis de publicaciones internacionales y en internet, la mayor parte de las cuales han sido editadas por organizaciones gubernamentales y no gubernamentales, a partir de los proyectos en que han participado. En este sentido, destacan organismos de la ONU como el Fondo de Desarrollo de las Naciones Unidas para la Mujer (UNIFEM) y ONU Mujeres, y las etapas por las que ambos atravesaron ${ }^{5}$ implicaron ciertos cambios significativos: en una primera fase hubo una gran actividad en proyectos de PSG que se plasmó en la publicación de numerosos informes; en la segunda, ya desde ONU Mujeres, se evidenció una clara merma en la cantidad de pro-

articula estructuras de poder y diferencia. Para ampliarlo, véase Joan W. Scott, "Gender: A Useful Category of Historical Analysis", en Joan W. Scott, Gender and the Politics of History, Nueva York, Columbia University Press, 1999, pp. 28-50.

${ }^{5}$ El UnIFEM (Fondo de Desarrollo de las Naciones Unidas para la Mujer) se creó en 1985 para apoyar iniciativas para la mejora de la calidad de vida de las mujeres. En 2011 se fusionó con ONU Mujeres. 
yectos - al menos como promotores-, lo que dio paso a un mayor liderazgo de las organizaciones civiles.

Los presupuestos sensibles al género: estado de la cuestión teórico-metodológica

Los debates iniciales sobre los PSG en el mundo han rebatido dos presunciones: una, que las políticas públicas pueden ser neutras; la otra, que las asignaciones presupuestarias se basan en un reparto (mejor o peor, más equitativo o menos) que repercute de modo diferente en los distintos grupos socioeconómicos, pero no en categorías como las de ser hombre o ser mujer. Como indica Cagatay, ${ }^{6}$ los presupuestos no son documentos financieros sino políticos que reflejan valores y prioridades de una sociedad y las relaciones de poder que subyacen a ésta.

Para abordar este tema resulta necesario ubicarse en un marco comprensivo alejado del modelo económico clásico, de modo que la supuesta neutralidad de los presupuestos quede al descubierto. Efectivamente, en el contexto occidental, la ciencia económica se ha ido desarrollando según parámetros basados en coherencia lógica, modelos de elección individual y libre, y con referentes predominantemente masculinos. ${ }^{7}$ Este enfoque clásico se asienta en tres supuestos: el primero es un concepto de trabajo restringido; el segundo, una noción de eficacia económica basada en el producto nacional bruto/producto interior bruto y en el Sistema de Cuentas Nacionales, y el tercero, una aproximación cuantitativa que se aprecia en el predominio de técnicas estadísticas. Estos pilares se ponen en entredicho en la década de 1970, a partir de la corriente renovadora del feminismo que reelabora y redefine conceptos, ámbitos y temáticas. Una noción clave ha sido la de trabajoactividad, que permitió desarrollar tipologías más inclusivas

${ }^{6}$ Nilüfer Cagatay, Mümtaz Keklik, Radhika Lal y James Lang, Budgets as if People Mattered: Democratizing Macroeconomic Policies, Social Development and Poverty Elimination Division Conference Paper Series, núm. 4, s.l., United Nations Development Programme, mayo de 2000.

${ }_{7}$ Una obra clave es la de Marianne Ferber y Julie Nelson, Más allá del hombre económico, Madrid, Cátedra, 2004. 
que tenían en cuenta las percepciones y el universo simbólico asociado a este concepto: los usos del tiempo, la simultaneidad en múltiples tareas, la creación de valor, estereotipos laborales, etcétera. ${ }^{8}$ Los principales tipos de trabajos analizados han sido el remunerado/no remunerado y el formal/informal. ${ }^{9}$ Como se demuestra en numerosas investigaciones, este tipo de trabajos y sectores se estructura de modo diferente en los diversos contextos mundiales de dominación. En el caso africano, se hace evidente el peso del trabajo informal, la compleja articulación del resto de los sectores mencionados y el papel de las mujeres en las estrategias de sobrevivencia e imbricación entre tipos de trabajo y sectores. ${ }^{10}$

En síntesis, un enfoque de género implica la reflexión sobre conceptos como desarrollo bumano y la ampliación de otros, como eficacia, más allá de lo meramente económico. ${ }^{11}$ Toda esta tarea de redefinición se hace especialmente relevante en contextos no occidentales, donde los estereotipos culturales se interconectan -de modo complejo-con otros vértices, como género, etnia y estructura social.

Así pues, este campo de reciente investigación ha tenido una rápida evolución que se muestra en el cambio de conceptos, ámbitos de análisis y diseño de herramientas, y es objeto de intensos debates. ${ }^{12}$ De las experiencias desarrolladas destacan las de Australia, Reino Unido, países nórdicos, Canadá, algunos

${ }^{8}$ Theodore Greenstein, "Economic Dependence, Gender and the Division of Labour in the Home: A Replication and Extension", Journal of Marriage and Family, vol. 62 , núm. 2, mayo de 2000, pp. 322-335.

${ }^{9}$ Fondo de Desarrollo de las Naciones Unidas para la Mujer, El progreso de las mujeres en el mundo 2000, Nueva York, UNIFEM, 2000; y El progreso de las mujeres en el mundo 2008-2009, Nueva York, UNIFEM, 2008.

${ }^{10}$ Véanse Letty Chiwara (ed.), Changing Lives in Africa 2012, Nueva York, United Nations Entity for Gender Equality and the Empowerment of Women, 2013; y United Nations Entity for Gender Equality and the Empowerment of Women, World Survey on the Role of Women in Development, 2014. Gender Equality and Sustainable Development, Nueva York, Naciones Unidas, 2014; así como estudios temáticos como el de Olutoyin Mejiuni, Women and Power. Education, Religion and Identity, Dakar, Council for the Development of Social Science Research in Africa, 2013.

${ }^{11}$ Véanse obras de Amartya Sen como Elección colectiva y bienestar social, Madrid, Alianza Editorial, 2007.

${ }^{12}$ Economic Commission for Latin America and the Caribbean, Tools and Indicators for Gender Impact Analysis, Monitoring and Evaluation, Nueva York, Economic Commission for Latin America and the Caribbean, febrero-marzo de 2002. 
países latinoamericanos y otros de la zona de la Commonwealth. ${ }^{13}$

Las evaluaciones de gran parte de estas experiencias insisten en la repercusión de herramientas de trabajo como los indicadores sensibles al género ${ }^{14}$ y las evaluaciones de todo el proceso.

\section{La experiencia africana en presupuestos sensibles al género}

En el contexto africano, las iniciativas sobre PSG provienen de diversos agentes, si bien los proyectos de cobertura nacional han surgido de organismos internacionales, con participación de los gobiernos nacionales y, dependiendo del contexto, de la sociedad civil. UNIFEM, como entidad de las Naciones Unidas para la igualdad de género y el empoderamiento de las mujeres, ha sido el organismo más dinámico en los últimos decenios, hasta su fusión en ONU Mujeres en 2011. El programa más destacado ha sido el de Integración de los presupuestos sensibles al género en la agenda de la ayuda internacional, fundado desde la Comisión Europea en 2008 y que implicaba tanto la investigación como la asistencia técnica o capacitación, con una duración de tres años y con el objetivo de mostrar el impacto de los PSG en la equidad de género. En la primera fase participaron países africanos que, en su mayoría, ya tenían experiencias locales y que han sido, con posterioridad, difusores de esta perspectiva.

Los seis países africanos que aquí se analizan han sido pioneros en PSG, aunque con diversa intensidad ${ }^{15} \mathrm{y}$ ámbitos de ac-

${ }^{13}$ Experiencias descritas en Debbie Budlender, "La economía política de los presupuestos de las mujeres en el sur", en Rosalba Todaro y Regina Rodríguez (eds.), El género en la economía, Santiago de Chile, Isis Internacional, 2000, pp. 39-60; Commonwealth Secretariat, Gender Budget Initiative: A Commonwealth Initiative to Integrate Gender into National Budgetary Processes, Londres, Commonwealth Secretariat, 1999.

${ }^{14}$ Véase Siobhan Austen, Therese Jefferson y Vicki Thein, "Gendered Social Indicators and Grounded Theory”, Feminist Economics, vol. 9, núm. 1, 2003, pp. 1-18.

${ }^{15}$ United Nations Development Fund for Women, UNIFEM's Work on GenderResponsive Budgeting. Evaluation Report, Nueva York, UNIFEM, 2010. 
tuación. Como se aprecia en el cuadro, los actuales indicadores globales visibilizan diferencias entre estos países, si bien hay un corte entre los dos con rentas más elevadas (Sudáfrica y Marruecos) y el resto; sin embargo, esto no siempre se refleja en otros índices, como, por ejemplo, en el de igualdad de género.

Datos de los seis países africanos pioneros en PSG (2015)

\begin{tabular}{|c|c|c|c|c|c|}
\hline & $\begin{array}{c}\text { Población } \\
\text { total/ } \\
\text { (\% de } \\
\text { mujeres) }\end{array}$ & $\begin{array}{l}\text { Renta } \\
\text { per } \\
\text { cápita }\end{array}$ & $\begin{array}{c}\text { PIB } \\
\text { (dólares) }\end{array}$ & $\begin{array}{c}\text { Índice } \\
\text { de } \\
\text { desarrollo } \\
\text { bumano }\end{array}$ & $\begin{array}{c}\text { Indice de } \\
\text { igualdad } \\
\text { género } \\
\text { en África. } \\
\text { Puntos y } \\
\text { puesto } \\
\text { de } 52\end{array}$ \\
\hline Sudáfrica & $\begin{array}{c}52.8 \\
\text { millones } \\
(51.46 \%)\end{array}$ & 7.610 & $\begin{array}{c}335.4 \\
\text { billones }\end{array}$ & 121 & $\begin{array}{c}74.5 \\
1^{\text {o }}\end{array}$ \\
\hline Marruecos & $\begin{array}{c}33 \\
(50.64 \%)\end{array}$ & 2.960 & 107.6 & 130 & $\begin{array}{c}52.9 \\
26^{\circ}\end{array}$ \\
\hline Mozambique & $\begin{array}{c}25.8 \\
(51.11 \%)\end{array}$ & 510 & 16.3 & 185 & $\begin{array}{c}61.9 \\
8^{a}\end{array}$ \\
\hline Ruanda & $\begin{array}{c}11.8 \\
(51.17 \%)\end{array}$ & 600 & 6.7 & 167 & $\begin{array}{c}74.3 \\
2^{\circ}\end{array}$ \\
\hline Tanzania & $\begin{array}{c}49.3 \\
(49.99 \%)\end{array}$ & 570 & 31.5 & 152 & $\begin{array}{c}64.2 \\
16^{a}\end{array}$ \\
\hline Uganda & $\begin{array}{c}37.6 \\
(49.87)\end{array}$ & 440 & 23.5 & 161 & $\begin{array}{c}63.4 \\
12^{\circ}\end{array}$ \\
\hline
\end{tabular}

Fuente: African Development Bank Group, “Areas of Special Emphasis”, www. afdb.org, s.f. [<https://www.afdb.org/en/about-us/mission-strategy/areas-of-specialemphasis/ > , consultado el 1 de marzo de 2017.] Elaboración propia.

Aspectos comunes a la mayor parte de estos países son la relevancia de los movimientos de mujeres, ${ }^{16}$ el aumento del

${ }^{16}$ Véanse Akouma Adomako y Signe Arnfred, African Feminist Politics of Knowledge. Tensions, Challenges, Possibilities, Uppsala, Nordiska Afrikan Institutet, 2009; Carmen Ascanio Sánchez, "Feminismos africanos", en Elena Hernández Corrochano (ed.), Teoría feminista y antropología: claves analíticas, Madrid, Editorial Ramón Areces, 2012, pp. 177-198; Amina Mama y Margo Okazawa-Rey, "Militarism, 
interés por parte de los académicos y la inclusión de estos temas en las agendas gubernamentales. Esto es evidente en las trayectorias de los presupuestos sensibles al género.

\section{Sudáfrica}

En la actualidad, este país ocupa el primer puesto del índice de igualdad de género a partir de una trayectoria continuada de trabajo en esta dirección, liderada primero desde la sociedad civil y rápidamente asumida desde las políticas públicas. El desarrollo de los principales programas implantados ha recibido una significativa aportación económica de las agencias de desarrollo externas, algo que - por otra parte- es generalizable a la mayor parte de estas experiencias en África.

El país desarrolló el primer enfoque de PSG en 1994, promovido desde la sociedad civil y continuado desde el gobierno posterior al apartheid. En ese periodo se pusieron en marcha iniciativas para impulsar la mejora de las condiciones de vida de la población en general y de las mujeres en particular. Debido a las limitaciones de presupuesto, la prioridad fue analizar la realidad cotidiana de las mujeres como indicador de las posibles mejoras que se introducirían. Uno de los aspectos más interesantes de los programas desarrollados en Sudáfrica fue incluir la interseccionalidad atendiendo asuntos de género, clase y etnia (raciales) y mostrando cómo pueden y deben ser incorporadas otro tipo de categorías a estos enfoques. ${ }^{17}$

En las evaluaciones realizadas en la primera fase de experiencias PSG, se hacen varias recomendaciones, ${ }^{18}$ entre las que destaca la necesidad de analizar el aporte no remunerado a la economía del cuidado que realizan mayoritariamente las muje-

Conflict and Women's Activism”, Feminist Africa, vol. 10, agosto de 2008, pp. 1-8; United Nations Development Fund for Women, Gender Responsive Budgeting and Aid Effectiveness: Knowledge Briefs, Nueva York, UNIFEM, 2009.

${ }_{17}$ United Nations Development Fund for Women, Engendering Budgets: The Southern African Experience. Report of a UNIFEM Regional Workshop, Harare, Zimbabwe, 9-10 de noviembre de 1998.

${ }^{18}$ Debbie Budlender y Guy Hewitt, Engendering Budgets. A Practitioners' Guide to Understanding and Implementing Gender-responsive Budgets, Melbourne, Commonwealth Secretariat, 2003. 
res. Basándose en estos diagnósticos, el Ministerio de Finanzas ha liderado los cambios en el último decenio a través de dos programas: ${ }^{19}$ uno basado en la creación de políticas de trabajo decente focalizado en igualdad de género y otro centrado en la seguridad alimentaria y dirigido a reducir los altos niveles de pobreza. A través de este último, se han llevado a cabo acciones como la de cero tasas, que incide en los precios de los alimentos básicos, en el papel de las mujeres como sostenedoras de hogares y en la baja de tarifas en servicios básicos como el agua, la electricidad y el cuidado infantil. Gran parte de estas medidas tienen su reflejo en los PSG, con el foco de atención puesto en los vínculos entre sociedad civil, Parlamento y gobierno, algo que se ha mantenido durante la trayectoria en estas experiencias.

\section{Marruecos}

El liderazgo desde las políticas públicas es aquí predominante, si bien los resultados han sido dispares en diferentes áreas de desarrollo. De hecho, Marruecos, a pesar de sus positivos indicadores de renta e índice de desarrollo humano, ocupa el puesto 26 (entre 52 países africanos) en el índice de igualdad de género. Esto se debe a que ha invertido de modo relevante en áreas como salud y educación, pero ha relegado otras, como las relacionadas con la igualdad de oportunidades económicas, legislativas y de participación en las instituciones.

Los iniciales planteamientos de PSG se desarrollaron en el Plan de Desarrollo Económico (2000-2004), que explicitaba las prioridades del presupuesto nacional; sin embargo, su enfoque era macroeconómico, sin atender al género, que sólo en los últimos años se ha abordado de modo transversal. De hecho, apenas desde 2007 se ha incluido en los presupuestos del Estado una carta de instrucción a los ministerios donde se recomienda

${ }_{19}$ Patricia Nyman, "Experience with Gender Budgeting from South Africa", en Association of German Development NGOs y Prospects for Africa-Europe's Policies, Gender Budgeting and Democratic Governance: Experience from Africa and Europe, Bonn, Association of German Development NGOs y Prospects for Africa-Europe's Policies, 2010, pp. 8-9. 
la inclusión de la perspectiva de género en sus presupuestos y se especifica la utilidad de incluir objetivos e indicadores sensibles al género.

Desde la cooperación exterior, el marco utilizado en Marruecos se ha basado en la Iniciativa Nacional para el Desarrollo Humano, cuyos objetivos principales son la reducción de la pobreza, la exclusión y la vulnerabilidad social, a través del fortalecimiento de capacidades y de un mejor acceso a servicios e infraestructuras. La ayuda oficial al desarrollo ha sido importante para estos proyectos, ya que ha constituido, en los diferentes periodos, alrededor de $6 \%$ del presupuesto nacional. UNIFEM desde el 2001, y ONU Mujeres después, han apoyado de modo continuado esta labor a través de los presupuestos generales del Estado. En una primera fase se implantó un programa liderado por la Dirección de Presupuesto del Ministerio de Economía y Finanzas, el cual pretendía fortalecer capacidades y desarrollar herramientas y metodologías de los PSG. Se elaboró un manual para presupuestos y una guía para parlamentarios y organizaciones no gubernamentales. En la segunda fase, a los primeros organismos se unieron otras direcciones del Ministerio de Economía y Finanzas, y se introdujo la herramienta de los informes de género, que ha sido adoptada por numerosos departamentos para examinar sus políticas y estrategias y aportar información sobre el gasto relacionado con el género. La mayor atención se ha centrado en el sector educativo y la alfabetización y las mujeres han sido las principales beneficiarias de estos programas $(80 \%)$, especialmente en las áreas urbanas.

Entre las recomendacione ${ }^{20}$ para este país se apuntan: continuar la colaboración entre donantes de ayuda y ministerios clave; evaluar las estrategias sectoriales y los indicadores de género; identificar las prioridades por regiones, provincias y comunas $y$, por último, desarrollar herramientas y enfoques sensibles al género.

${ }^{20}$ Nalini Burn, Integrating Gender Responsive Budgeting into the Aid Effectiveness Agenda: Morocco Country Report, Nueva York, United Nations Development Fund for Women, 2008. 


\section{Mozambique}

Las iniciativas en este país parten del gobierno y se agrupan en los Planes Quinquenales, que se apoyan tanto en los presupuestos generales del Estado como en los programas de PSG comenzados por UNIFEM en 2003. Las agencias internacionales de cooperación han apoyado a sectores clave para el desarrollo y, de hecho, los donantes han firmado un Memorando de Entendimiento con el gobierno para desarrollar estas herramientas, acuerdo que se evalúa anualmente. Por otra parte, se han establecido mecanismos de coordinación conjunta; por ejemplo, un Grupo de Coordinación de Género que tiene representantes del gobierno, donantes y sociedad civil para procesos de revisión. ${ }^{21}$

Los primeros programas desarrollados por UNIFEM han tenido varias fases. Según la evaluación de Holvgoet e Inberg, ${ }^{22}$ en la primera se desarrollaron aspectos relacionados con las capacidades, especialmente de monitoreo. En la segunda se pasó a enfoques centrados en los propios actores políticos, con la participación de los Ministerios de Planificación y el de Finanzas. Los programas se han orientado por áreas temáticas, como violencia contra las mujeres (VAW por sus siglas en inglés) y salud materna, que han generado un programa nacional para mejorar los servicios de salud. Este sector se eligió como prioritario para implementar el análisis de PSG y en su evaluación se apreció un fuerte "sesgo maternal" en las indicaciones señaladas para el género, con focos de atención en mortalidad materna $\mathrm{y}$ en $\mathrm{VIH} /$ sida entre mujeres gestantes y en edad reproductiva.

La evaluación incluye cuatro recomendaciones: que los grupos y programas relacionados con PSG colaboren con otros relacionados con las cuentas nacionales, como los grupos de análisis de presupuestos, pobreza, etcétera; traducir prioridades y actividades de género en indicadores y planes; desarrollar debates sobre el manejo de las finanzas públicas, el fortaleci-

${ }^{21}$ Teresa Buchen, Gender Budget Initiatives, Uganda, Mozambique and Nicaragua, Viena, Vienna Institute for International Dialogue and Cooperation, 2007.

${ }_{22}$ Nathalie Holvgoet y Liesbeth Inberg, Integrating Gender Responsive Budgeting into the Aid Effectiveness Agenda: Mozambique Country Report, Nueva York, United Nations Development Fund for Women, 2008. 
miento de capacidades y la asistencia técnica; y, por último, sugerir a los donantes que trabajen e inviertan en la adaptación de sus lineamientos generales (ya elaborados y aplicados) a los contextos y realidades concretos del país.

\section{Ruanda}

Este país ha tenido una trayectoria que habría sido impensable dos décadas atrás. En la actualidad ocupa el segundo puesto en el índice de igualdad de género, con un importante equilibrio entre las diferentes áreas por desarrollar, a pesar de su baja ubicación en el índice de desarrollo humano. La primera iniciativa de PSG se dio en 2002 como parte de un programa más amplio de transversalidad de género dentro del Ministerio de Género y apoyado por agencias internacionales. En el plano estatal se implementaron medidas de pilotaje en cinco ministerios (Educación, Agricultura y Ganadería, Salud, Agua y recursos naturales y Gobierno local y asuntos sociales), cuyo personal recibió la capacitación necesaria. Dicho programa finalizó en 2004, y una evaluación mostró un impacto mínimo del enfoque de género.

Con posterioridad, la guía básica para el mejoramiento del país ha sido la denominada Estrategia para la Reducción de la Pobreza, desarrollada de 2008 a 2012 y que incluye tres vértices clave: el crecimiento sostenible para el empleo y las exportaciones, el desarrollo rural integral y la gobernabilidad. Esta estrategia implica temas transversales como género, $\mathrm{VIH} / \mathrm{sida}$, medioambiente, inclusión social y juventud, desarrollados a partir de gastos a mediano plazo. Los mecanismos de coordinación se basan en la consulta conjunta y en el diálogo entre el gobierno, los donantes y la sociedad civil, que conforman grupos para revisar colectivamente la planificación estratégica y los presupuestos.

En este país habría que notar la considerable aportación de la ayuda oficial al desarrollo, que ha supuesto $24 \%$ del PIB nacional en el último decenio. En 2008 se lanzó un programa de UNIFEM con el objetivo de fortalecer las capacidades institucionales para elaborar informes, análisis de género, recopi- 
lación de datos y monitoreo. Los cuatro sectores básicos para su desarrollo han sido educación, salud, infraestructura y agricultura. Entre las recomendaciones se plantean cinco puntos básicos que se implementan en la actualidad. ${ }^{23}$ El primero es la necesidad de crear un marco institucional que apoye el monitoreo y la coordinación para la implantación de los PSG. En segundo lugar, las reformas del sector público y de la gestión de las finanzas públicas deberán ser el punto de entrada de los PSG. Tercero, hay que fortalecer las capacidades internas de las instituciones, tanto local como nacionalmente. Cuarto, las evaluaciones son imprescindibles. Por último, se deben desarrollar mecanismos de rendición de cuentas entre el gobierno y los socios externos.

\section{Tanzania}

Este país pionero en los PSG ha tenido importantes altibajos en su implantación y, en especial, vínculos débiles entre las políticas públicas y las organizaciones ciudadanas. El enfoque de PSG comenzó en 1997 con el Programa Tanzano de Redes de Género en cooperación con la Alianza Feminista Activista (FemAct), que ha atendido el fortalecimiento de capacidades, la integración del enfoque de género en las diferentes etapas de planificación y presupuestación, la investigación y el análisis de políticas, concientización y difusión, el diálogo con los donantes, y otros. Sin embargo, las medidas transversales se han introducido en el último decenio a través de los presupuestos generales, por lo que se carece aún de guías o explicaciones específicas sobre el diseño y la ejecución de presupuestos sensibles al género. De hecho, una de las últimas evaluaciones realizadas apunta como debilidad que el gobierno apenas informa sobre presupuestos y finanzas, y lo atribuye a la escasa participación social en estos procesos. ${ }^{24}$

${ }^{23}$ John Mutamba y Cyma Mbayiha, Integrating Gender Responsive Budgeting into the Aid Effectiveness Agenda: Rwanda Country Report, Nueva York, United Nations Development Fund for Women, 2008.

${ }^{24}$ United Nations Entity for Gender Equality and the Empowerment of Women, World Survey on the Role of Women in Development, op. cit. 
En cuanto al monitoreo del proceso presupuestario, desde 2007-2008 las instituciones del Estado están obligadas a informar detalladamente sobre los planes estratégicos y el marco político nacional para el desarrollo mediante la Estrategia Nacional para el Crecimiento y la Reducción de la Pobreza (conocida como MKUKUTA en swahili), que tiene tres áreas prioritarias: crecimiento y reducción de la pobreza en términos de ingresos; mejoramiento de la calidad de vida y el bienestar social, y gobernabilidad y rendimiento de cuentas. Los temas de género se encuentran integrados transversalmente en las prioridades de esta estrategia, desde la que se han creado 84 indicadores desagregados por zona geográfica, zona rural/ urbana, sexo y nivel de pobreza.

Un monto importante del presupuesto del gobierno tanzano proviene de la ayuda oficial al desarrollo (alrededor de $30 \%$ en los dos últimos decenios); de hecho, los donantes más relevantes trabajan con una Estrategia Conjunta de Asistencia que cuenta con varias estructuras paralelas de diálogo y alineamiento con organismos del gobierno. Los denominados Grupos de Socios para el Desarrollo, una de esas estructuras, se basan en temáticas prioritarias. Uno de ellos, por ejemplo, se ocupa de la igualdad de género y da seguimiento a la evolución de estas líneas en los sectores y organismos pertinentes, aunque no suelen tener representación directa en organismos encargados de decisiones económicas. Los sectores principales son: educación, salud, agricultura, agua y gobernabilidad local. De especial interés ha sido la trayectoria investigadora de los diferentes programas, que incluyen política tributaria, hogares con jefatura femenina, atención de las mujeres al viH/ sida en los hogares, etcétera.

Entre las principales recomendaciones se plantean: ${ }^{25}$ mejorar las instrucciones para priorizar las líneas presupuestarias y, en especial, cómo desarrollar los objetivos para avanzar en los PSG; perfeccionar aspectos estadísticos relacionados con la desagregación de datos por sexo, así como la inclusión y la eva-

${ }^{25}$ Liisa Kytölä, Integrating Gender Responsive Budgeting into the Aid Effectiveness Agenda: Tanzania Country Report, Nueva York, United Nations Development Fund for Women, 2008. 
luación de indicadores de género en todos los programas y organismos, y por último, se alienta a incrementar y mejorar la relación entre los donantes internacionales y los actores - públicos y privados- relacionados con el género.

\section{Uganda}

Los proyectos sobre presupuestos de género comenzaron en 1997 por parte de organizaciones no gubernamentales, aunque los PSG arrancaron en 1999 gracias a la coalición de mujeres activistas y parlamentarios. ${ }^{26}$ El Foro para las Mujeres en la Democracia ha sido clave, ya que han luchado por la introducción de presupuestos participativos y el monitoreo en diferentes niveles de las políticas públicas. Desde este Foro se han realizado tareas de investigación, evaluación y apoyo a sectores clave del país, como agricultura, salud, educación, agua y saneamiento, justicia, policía y seguridad. Al mismo tiempo, han promovido la capacitación de legisladores, planificadores, funcionarios, etcétera. El Ministerio de Finanzas, Planificación y Desarrollo Económico ha participado activamente en la entrada de los PSG desde 2003-2004, al incluirlos en las instrucciones para elaborar presupuestos, al tiempo que colaboraba estrechamente con el Ministerio de Género, Trabajo y Desarrollo Social.

El marco político para el desarrollo en este país se ha basado en el Plan de Acción para la Erradicación de la Pobreza (PEAP) (2004-2005 y 2007-2008). En su inicial análisis apunta a la desigualdad de género como una de las barreras para erradicar la pobreza y a la necesidad de integrar este enfoque a los sectores más importantes de desarrollo del país. La base para ejecutar el PEAP desde la cooperación al desarrollo ha sido la Estrategia Conjunta de Asistencia en Uganda, 2005-2009. Desde ahí se compromete a los donantes a participar en actividades relacionadas con estos temas: apoyar la ejecución de la Política de Género de Uganda y promover la equidad de género en la vida familiar, laboral y comunitaria, y en temáticas relacionadas con la vulnerabilidad de las mujeres. Recientemente se trabaja

${ }^{26}$ Buchen, Gender Budget Initiatives, Uganda, Mozambique and Nicaragua, op. cit. 
con un marco conjunto de evaluación como estrategia innovadora. Los mecanismos de coordinación se basan en un grupo de apoyo presupuestario de donantes, dentro del que hay un Grupo de Coordinación de Género, constituido en 2001 con el objetivo básico de promover la igualdad de género y el empoderamiento de las mujeres. Está abierto a representantes de organizaciones donantes, tanto bilaterales como multilaterales, $\mathrm{y}$ a las organizaciones no gubernamentales internacionales. Su estrategia básica parte de transversalizar el género en políticas y programas nacionales, enlazándose con otros grupos. En 2008, el PEAP fue remplazado por un plan nacional de desarrollo que ha mostrado dudas respecto al aprovechamiento de la experiencia anterior, de las buenas prácticas y de la transversalidad de género.

Como sucede en gran parte de los países reseñados, Uganda recibe una importante ayuda exterior, que en el periodo 2008 2009 supuso $30 \%$ del presupuesto, ${ }^{27}$ con índices similares en la actualidad. Todos los donantes utilizan el apoyo directo al presupuesto y a proyectos, generalmente a través del Crédito para Apoyar la Reducción de la Pobreza del Banco Mundial, organismo que es su principal donante en la actualidad.

Entre las recomendaciones para este país se encuentran: ${ }^{28}$ introducir los PSG en los nuevos planes de desarrollo; estimular el papel del grupo de Coordinación de Donantes para evaluar planes y programas y para incentivar la participación de los diferentes actores; incluir la capacitación en materia de PSG en todos los niveles de lo público y la sociedad civil para formar funcionarios relacionados con el presupuesto; incrementar las auditorías de género para evaluar las iniciativas de PSG; $y$, por último, incentivar un papel más activo por parte de los diferentes ministerios que asegure su transparencia, eficiencia y eficacia.

${ }^{27}$ Nite Tanzarn, Integrating Gender Responsive Budgeting into the Aid Effectiveness Agenda: Uganda Country Report, Nueva York, United Nations Development Fund for Women, 2008.

${ }^{28}$ Mary Kuzambiza, A Case Study of Gender Responsive Budgeting in Uganda, Londres, Commonwealth Secretariat, 2013. 
Otros países

La experiencia de estos países pioneros ha influido en los recientes proyectos de PSG en otros contextos africanos, introduciendo nuevos enfoques y temáticas. Ejemplos de interés ${ }^{29}$ han sido los de Camerún, que ha puesto el foco de atención en la infraestructura del transporte y los beneficios de las mujeres en materia de accesibilidad; Etiopia, Cabo Verde, Senegal y Zimbabue, donde ha habido una importante movilización por parte de ministerios, especialmente en áreas de salud y educación. En todo caso, en esta última etapa resulta relevante el menor presupuesto desde ONU Mujeres y ciertas resistencias a implantar la transversalidad de género que han paralizado propuestas e intervenciones. ${ }^{30}$

\section{Comparación de experiencias}

Una mirada al contexto africano muestra que las condiciones de mujeres y hombres son muy diferentes en múltiples áreas, como educación, trabajo-empleo, participación, salud, etcétera. En los países seleccionados, los datos macroeconómicos y los análisis muestran realidades distintas. Por ejemplo, respecto al nivel de rentas y el PIB, por una parte tenemos países como Sudáfrica y Marruecos, y, por otra, el resto. Sin embargo, desde un enfoque más cualitativo, resulta evidente que la falta de correlación entre índices de desarrollo y de igualdad

${ }^{29}$ Véanse los informes de Dorothy Adebanyo, Gender Budgeting: Case Study of the Zimbabwe Experience, Open Society Initiative for Southern Africa, s.f.; Republic of Cabo Verde-Cape Verdean Institute for Gender Equality and Equity, Cabo Verde's Report: Beijing +20 on the Implementation of the Beijing Declaration and Platform for Action, Praia, junio de 2014; Jacinta Muteshi y Tirowork Tizazu, Integrating Gender Responsive Budgeting into the Aid Effectiveness Agenda: Ethiopia Country Report, Nueva York, United Nations Development Fund for Women, 2008; Bruno Ntakeu y Fon Mercy, Integrating Gender Responsive Budgeting into the Aid Effectiveness Agenda: Cameroon Country Report, Nueva York, United Nations Development Fund for Women, 2008.

${ }^{30}$ Fondo de Desarrollo de las Naciones Unidas para la Mujer, El progreso de las mujeres en el mundo 2008-2009, op. cit.; Chiwara (ed.), Changing Lives in Africa 2012, op. cit.; y United Nations Entity for Gender Equality and the Empowerment of Women, World Survey on the Role of Women in Development, op. cit. 
de género expresa la necesidad de redefinir conceptos como desarrollo humano, eficacia económica, y otros, incluidos indicadores cualitativos y con perspectiva de género sobre estas realidades.

El enfoque de los PSG ha estado presente en el diseño de estos planes y programas, pero la evaluación muestra que la transversalidad de género no siempre ha sido la estrategia de actuación. Por tanto, en los seis países se aprecian enfoques diferentes en los planes, programas y proyectos, aunque tres elementos se repiten con distinta intensidad: el importante papel de los gobiernos nacionales; la integración de los PSG a los principales planes y estrategias de desarrollo de cada país, y su vinculación con la sociedad civil y la ayuda externa (ayuda oficial al desarrollo).

Ciertamente, en los países analizados las experiencias en PSG han sido desarrolladas mayoritariamente desde las políticas públicas, aunque con diferentes vínculos con otros agentes sociales. En países como Sudáfrica, Tanzania y Uganda, las iniciativas desde la sociedad civil, especialmente de asociaciones de mujeres, han tenido un gran protagonismo y un continuo seguimiento de las experiencias desarrolladas. Esta fuerte vinculación entre políticas gubernamentales y sociedad civil parece incidir de modo potente en enfoques transversales y participativos. El apoyo a los PSG se suele apreciar en su integración a programas presupuestarios y ministerios más relevantes, como los de Economía, Hacienda/Finanzas, y a los marcos políticos más significativos para el desarrollo de cada país. Por ejemplo, la Estrategia Nacional para el Crecimiento y la Reducción de la Pobreza en el caso de Tanzania, planes estratégicos como ocurre en Ruanda, o a través de los presupuestos generales.

Con todo, no hay que olvidar el papel de las agencias de cooperación y la ayuda externa. De hecho, los objetivos de los PSG en cada contexto se relacionan con agendas externas, que coinciden en ciertas prioridades: potenciar la democracia, la participación de la sociedad civil, la reducción de la pobreza y, por supuesto, la rendición de cuentas. ${ }^{31}$ Por esa razón, la ma-

${ }^{31}$ Janet Stotsky, Lisa Kolovich y Suhaib Kebhaj, Sub-Saharan Africa: A Survey of Gender Budgeting Efforts, IMF Working Paper 16/152, Washington, International Monetary Fund, julio de 2016. 
yor parte de los proyectos prioriza las áreas que consideran clave para estos asuntos: formación/educación, salud, planes contra la pobreza/exclusión, etcétera. Sin embargo, también se aprecian diferencias que parecen depender de complejas interrelaciones y dependencias entre, por una parte, la ayuda externa ${ }^{32}$ y la conformación de la agenda de desarrollo y, por otra, el enfoque gubernamental sobre sus políticas y la participación de la sociedad civil. Esto explica las diferentes trayectorias de, por ejemplo, Sudáfrica, cuyo gobierno se ha vinculado estrechamente con la sociedad civil, y Marruecos, cuyas políticas gubernamentales han priorizado unas áreas de desarrollo frente a otras, con evidentes luces y sombras. Ciertamente, este último país es un caso paradigmático, ya que ha conseguido notables mejoras en áreas como sanidad y educación, pero ha relegado las relacionadas con la participación en el mercado laboral, aspectos legislativos e institucionales (representación política). Este desequilibrio se muestra en elevados índices de desarrollo en algunas áreas que contrastan con el bajo índice de igualdad de género.

En todo caso, las experiencias en PSG han atravesado dos fases: una inicial basada en investigaciones/estudios o informes previos o evaluaciones para conocer las realidades de cada contexto, y otra centrada en la creación de herramientas de intervención, como guías, recomendaciones, indicadores, etcétera. Estrategias repetidas en todos los países han sido la sensibilización de funcionarios de los gobiernos y la priorización de intervenciones en el ámbito nacional, relegando niveles como el provincial y el local.

\section{Recomendaciones y consideraciones finales}

Los informes y las evaluaciones realizadas, escasas para la relevancia de esta herramienta, han incidido en varios aspectos que sintetizamos como recomendaciones para futuras intervenciones:

${ }^{32} \mathrm{La}$ OCDE es el mayor donante para el África Subsahariana, con un total de 24000 millones de dólares en el 2015: < http://www.oecd.org/dac/>, consultado el 1 de marzo de 2017. 
- Apoyo estadístico. En la mayor parte de los casos se echa de menos la desagregación de datos en las estadísticas nacionales y el diseño de otras herramientas, como indicadores sensibles al género.

- Ampliar la cantidad y la calidad (cualitativo) de los estudios, tanto nacionales como locales. Esto resulta clave para tener una visión amplia de la realidad cotidiana, de los espacios de especial interés y de las prioridades regionales.

- Priorizar áreas de estudio e intervención debidamente justificadas. Un ejemplo es el aporte del trabajo no remunerado a la economía de cuidado.

- Incrementar el desarrollo de herramientas sensibles al género (guías, informes, manuales, etcétera) y de protocolos para la comparación de experiencias.

- Incentivar los debates sobre manejo de finanzas públicas, fortalecimiento de capacidades y PSG. Se hace imprescindible el trabajo conjunto de los grupos que lideren PSG y los departamentos relacionados con cuentas nacionales.

- Ampliar la colaboración entre donantes internacionales, gobiernos nacionales y organizaciones civiles. En este sentido, resulta clave implementar herramientas de coordinación y evaluación. Se sugiere también elaborar evaluaciones a profundidad sobre el papel de las agencias de desarrollo a mediano y largo plazos.

Es importante reiterar la relevancia del enfoque de género y su transversalidad en las políticas e intervenciones, así como el concepto de desarrollo humano. En segundo término, si se atiende a los índices de igualdad de género, las estrategias más exitosas - por la continuidad y asentamiento de políticas- $\mathrm{pa-}$ recen relacionarse con las experiencias que han vinculado políticas públicas, sociedad civil y otros actores relevantes; por lo tanto, resultan vitales herramientas de coordinación, participación y fortalecimiento de la sociedad civil. Por último, hay que destacar la necesidad de iniciar líneas de investigación en esta temática, tanto teóricas como empíricas, e incentivar la publicación de experiencias y evaluaciones, algo imprescindible para una labor de sistematización y comparación. 
Dirección institucional de la autora:

Centro de Estudios Africanos

Universidad de La Laguna

Facultad de Educación, Módulo B.

Apartado Postal 456

38200, San Cristóbal de La Laguna

Santa Cruz de Tenerife, España

\section{Bibliografía}

AdDabBo, Tindara, "Gender Budgeting in the Capability Approach. From Theory to Evidence", presentación invitada en la WiSE: Women in Scotland's Economy Series Conference 'Counting on Women-Gender, Care and Economics', Glasgow Caledonian University, 24-26 de mayo de 2011. [ < www.gcu.ac.uk/media/ gcalwebv2/theuniversity/centresprojects/wise/addabbo.pdf $>$, consultado el 5 de julio de 2016.]

Adebanyo, Dorothy, Gender Budgeting: Case Study of the Zimbabre Experience, Open Society Iniciative for Southern Africa, s.f. [<https://www.dgf.ug/sites/default/files/dgf_publications/ Gender\%20Budgeting\%20Case\%20Study\%20of\%20the\%20Zimbabwe\%20Experience.pdf > , consultado el 5 de julio de 2016.]

Adomako, Akouma y Signe Arnfred, African Feminist Politics of Knoreledge. Tensions, Challenges, Possibilities, Uppsala, Nordiska Afrikan Institutet, 2009.

African Development Bank Group, "Areas of Special Emphasis", wwr.afdb.org, s.f. [<https://www.afdb.org/en/about-us/ mission-strategy/areas-of-special-emphasis/ $>$, consultado el 1 de marzo de 2017.]

Ascanio SÁnchez, Carmen, "Feminismos africanos", en Elena Hernández Corrochano (ed.), Teoría feminista y antropología: claves analíticas, Madrid, Editorial Ramón Areces, 2012, pp. 177-198.

Ascanio SÁnChEZ, Carmen, "Gobernabilidad y presupuestos sensibles al género. Estado de la cuestión en África”, Pueblos. Revista de información y debate, 3 de agosto de 2012. [<www. revistapueblos.org/old/spip.php?article2487>, consultado en 16 de enero de 2017.]

AusTEN, Siobhan, Therese Jefferson y Vicki Thein, "Gendered Social Indicators and Grounded Theory", Feminist Economics, vol. 9, núm. 1, 2003, pp. 1-18. 
Buchen, Teresa, Gender Budget Initiatives, Uganda, Mozambique and Nicaragua, Viena, Vienna Institute for International Dialogue and Cooperation, 2007. [ < http://alt.vidc.org/fileadmin/Bibliothek/DP/pdfs/gbi_2007.pdf >, consultado en mayo de 2017.]

BuDLENDER, Debbie, "La economía política de los presupuestos de las mujeres en el sur", en Rosalba Todaro y Regina Rodríguez (eds.), El género en la economía, Santiago de Chile, Isis Internacional, 2000, pp. 39-60.

Budlender, Debbie y Guy Hewitt, Engendering Budgets. A Practitioners' Guide to Understanding and Implementing Gender-responsive Budgets, Melbourne, Commonwealth Secretariat, 2003.

Budlender, Debbie y Rhonda Sharp, con Kerri Allen, How to do a Gender-sensitive Budget Analysis: Contemporary Research and Practice, Canberra-Londres, Commonwealth Secretariat-Australian Agency for International Development, 1998. [<http:// femtech.blaugrau.at/fileadmin/downloads/Wissen/Themen/ Geld_regiert_die_Welt/How_to_do_a_gender-sensitive_budget_analysis.pdf $>$, consultado mayo de 2017.]

BuRN, Nalini, Integrating Gender Responsive Budgeting into the Aid Effectiveness Agenda: Morocco Country Report, Nueva York, United Nations Development Fund for Women, 2008. [< http://www2. unwomen.org/-/media/files/un\%20women/grb/resources/ integrating $\% 20$ gender $\% 20$ responsive $\% 20$ budgeting $\% 20$ into $\% 20$ the $\% 20$ aid $\% 20$ effectiveness $\% 20$ agenda-morocco $\% 20$ report. pdf?vs $=5955>$, consultado en mayo de 2017.]

Cagatay, Nilüfer, Mümtaz Keklik, Radhika Lal y James Lang, Budgets as if People Mattered: Democratizing Macroeconomic Policies, Social Development and Poverty Elimination Division Conference Paper Series, núm. 4, s.l., United Nations Development Programme, mayo de 2000. [<http://www.chs.ubc.ca/lprv/ PDF/lprv0482.pdf $>$, consultado en mayo de 2017.]

Chiwara, Letty (ed.), Changing Lives in Africa 2012, Nueva York, United Nations Entity for Gender Equality and the Empowerment of Women, 2013. [ < http://www.unwomen.org/-/ media/headquarters/attachments/sections/library/publications/2013/2/un-women-changing-lives-in-africa-2012\%20pdf. pdf?vs $=1834>$, consultado en mayo de 2017.]

Commonwealth Secretariat, Gender Budget Initiative: A Commonwealth Initiative to Integrate Gender into National Budgetary Processes, Londres, Commonwealth Secretariat, 1999. [<http://www2.unwomen.org/-/media/files/un\%20women/ grb/resources/a $\% 20$ commonwealth $\% 20$ initiative $\% 20$ to $\% 20$ 
integrate $\% 20$ gender $\% 20$ into $\% 20$ national $\% 20$ budgetary $\% 20$ processes.pdf? $v s=4713>$, consultado en mayo de 2017.]

Economic Commission for Latin America and the Caribbean, Tools and Indicators for Gender Impact Analysis, Monitoring and Evaluation, Nueva York, Economic Commission for Latin America and the Caribbean, febrero-marzo de 2002. [ <www.un.org/ womenwatch/ianwge/annualmeetings/2002/RepToolsndic. PDF > , consultado el 5 de julio de 2016.]

Ferber, Marianne y Julie Nelson, Más allá del hombre económico, Madrid, Cátedra, 2004.

Fondo de Desarrollo de las Naciones Unidas para la Mujer, El progreso de las mujeres en el mundo 2000, Nueva York, UNIFEM, 2000.

Fondo de Desarrollo de las Naciones Unidas para la Mujer, El progreso de las mujeres en el mundo 2008-2009, Nueva York, UNIFEM, 2008. Greenstein, Theodore, "Economic Dependence, Gender and the Division of Labour in the Home: A Replication and Extension", Journal of Marriage and Family, vol. 62, núm. 2, mayo de 2000, pp. 322-335.

HolVGOET, Nathalie y Liesbeth Inberg, Integrating Gender Responsive Budgeting into the Aid Effectiveness Agenda: Mozambique Country Report, Nueva York, United Nations Development Fund for Women, 2008.

KuZambiza, Mary, A Case Study of Gender Responsive Budgeting in Uganda, Londres, Commonwealth Secretariat, 2013. [< https:// consultations.worldbank.org/Data/hub/files/grb_papers_uganda_updf_final.pdf $>$, consultado en mayo de 2017.]

KүтӧLё̈, Liisa, Integrating Gender Responsive Budgeting into the Aid Effectiveness Agenda: Tanzania Country Report, Nueva York, United Nations Development Fund for Women, 2008.

Mama, Amina y Margo Okazawa-Rey, "Militarism, Conflict and Women's Activism", Feminist Africa, vol. 10, agosto de 2008, pp. 1-8.

MejIuni, Olutoyin, Women and Power. Education, Religion and Identity, Dakar, Council for the Development of Social Science Research in Africa, 2013.

Mutamba, John y Cyma Mbayiha, Integrating Gender Responsive Budgeting into the Aid Effectiveness Agenda: Rwanda Country Report, Nueva York, United Nations Development Fund for Women, 2008.

Muteshi, Jacinta y Tiruwork Tizazu, Integrating Gender Responsive Budgeting into the Aid Effectiveness Agenda: Ethiopia Country Report, Nueva York, United Nations Development Fund for 
Women, 2008. [ < http://www2.unwomen.org/-/media/files/ un $\% 20$ women/grb/resources/integrating\%20gender\%20responsive $\% 20$ budgeting\%20into $\% 20$ the $\% 20$ aid $\% 20$ effectiveness $\% 20$ agenda\%20-\%20 ethiopia\%20report.pdf?vs $=56>$, consultado en mayo de 2017.]

NTAKeu, Bruno y Fon Mercy, Integrating Gender Responsive Budgeting into the Aid Effectiveness Agenda: Cameroon Country Report, Nueva York, United Nations Development Fund for Women, 2008. [<http://www2.unwomen.org/-/media/files/ un $\% 20$ women/grb/resources/integrating\%20gender\%20responsive $\% 20$ budgeting $\% 20$ into $\% 20$ the $\% 20$ aid $\% 20$ effectiveness $\% 20$ agenda $\% 20$ cameroon $\% 20$ report.pdf?vs $=16>$, consultado en mayo de 2017.]

Nyman, Patricia, "Experience with Gender Budgeting from South Africa", en Association of German Development NGOs y Prospects for Africa-Europe's Policies, Gender Budgeting and Democratic Governance: Experience from Africa and Europe, Bonn, Association of German Development NGOs y Prospects for Africa-Europe's Policies, 2010, pp. 8-9. [ < http://venro.org/ uploads/tx_igpublikationen/2010_Afrika_EU_Gender_Budgeting_englisch.pdf $>$, consultado en mayo de 2017.]

Republic of Cabo Verde-Cape Verdean Institute for Gender Equality and Equity, Cabo Verde's Report: Beijing +20 on the Implementation of the Beijing Declaration and Platform for Action, Praia, junio de 2014. [ < http://www.uneca.org/sites/default/files/ uploaded-documents/Beijing20/NationalReviews/cape verde_beijing_review_report._english_version.pdf $>$, consultado en mayo de 2017.]

Sсотт, Joan W., "Gender: A Useful Category of Historical Analysis”, en Joan W. Scott, Gender and the Politics of History, Nueva York, Columbia University Press, 1999, pp. 28-50.

SEN, Amartya, Elección colectiva y bienestar social, Madrid, Alianza Editorial, 2007.

Sтотьку, Janet G., Lisa Kolovich y Suhaib Kebhaj, Sub-Saharan Africa: A Survey of Gender Budgeting Efforts, IMF Working Paper 16/152, Washington, International Monetary Fund, julio de 2016. [<https://www.imf.org/external/pubs/ft/wp/2016/ wp16152.pdf $>$, consultado en mayo de 2017.]

TANZARN, Nite, Integrating Gender Responsive Budgeting into the Aid Effectiveness Agenda: Uganda Country Report, Nueva York, United Nations Development Fund for Women, 2008. [< http:// www2.unwomen.org/-/media/files/un\%20women/grb/re- 
sources/integrating $\% 20$ gender $\% 20$ responsive $\% 20$ budgeting $\% 20$ into $\% 20$ the $\% 20$ aid $\% 20$ effectiveness $\% 20$ agenda-uganda $\% 20$ report.pdf? vs $=224>$, consultado en mayo de 2017.]

United Nations Development Fund for Women, Engendering Budgets: The Southern African Experience. Report of a UNIFEM Regional Workshop, Harare, Zimbabwe, 9-10 de noviembre de 1998.

United Nations Development Fund for Women, Gender Responsive Budgeting and Aid Effectiveness: Knowledge Briefs, Nueva York, UNIFEM, 2009. [http://iknowpolitics.org/sites/default/files/ knowledge20briefs20in20english.pdf, consultado en mayo de 2017.]

United Nations Development Fund for Women, UNIFEM's Work on Gender-Responsive Budgeting. Evaluation Report, Nueva York, UNIFEM, 2010. [ < http://www.unwomen.org/-/media/headquarters/media/publications/unifem/evaluation_grb_programme_en.pdf?vs $=2308>$, consultado en mayo de 2017.]

United Nations Entity for Gender Equality and the Empowerment of Women, World Survey on the Role of Women in Development, 2014. Gender Equality and Sustainable Development, Nueva York, Naciones Unidas, 2014. [http://www.unwomen. org/ /media/headquarters/attachments/sections/library/ publications/2014/unwomen_surveyreport_advance_16oct.pdf, consultado en mayo de 2017.] 
